\title{
INFLUÊNCIA DO COMPORTAMENTO IMITATIVO DE ADOLESCENTES E SEUS PARES NO USO DE SUBSTÂNCIAS PSICOATIVAS
}

\section{Influence of imitative behavior of adolescent and their peers in the use of psychoactive substances}

Fábio Cossa de Souza ${ }^{1}$, Luciane Bisognin Ceretta $^{1,2}$, Lisiane Tuon ${ }^{1,2}$, Priscyla Waleska Simões ${ }^{1,2}$, Graziela Amboni ${ }^{1}$

${ }^{1}$ Programa de Residência Multiprofissional em Atenção Básica/Saúde da Família Universidade do Extremo Sul Catarinense - UNESC, Criciúma. SC, Brasil.

2 Programa de Pós-Graduação em Saúde Coletiva (Mestrado Profissional) Universidade do Extremo Sul Catarinense - UNESC, Criciúma. SC, Brasil.

\section{Endereço para correspondência:}

Graziela Amboni

Programa de Residência Multiprofissional em Atenção Básica/Saúde da Família Universidade do Extremo Sul Catarinense - UNESC.

Avenida Universitária, 1105, Criciúma - SC

CEP - 88806-000

Email: gam@unesc.net 


\title{
Resumo
}

Esta pesquisa investigou a influência que o comportamento imitativo exerce na adoção de comportamentos relacionados ao uso de drogas em adolescentes de 12 a 17 anos. É caracterizada como um estudo observacional e transversal, de abordagem quantitativa, e foi realizada com 128 adolescentes de duas escolas públicas de Criciúma/SC. Os achados mostraram que muitos adolescentes possuem algum amigo que utiliza substâncias psicoativas e que muitos iniciaram o uso dessas substâncias mediante influência de uma amizade desse tipo. Em relação ao consumo de bebida alcoólica, 35,4\% dos estudantes afirmaram ter feito o uso por influência dos amigos, sendo mais predominante em indivíduos do gênero masculino; e 15,6\% afirmaram que usaram outras drogas pelo mesmo motivo. Observamos maior influência dos amigos no consumo de álcool entre os 15 e 17 anos ( $n=33 ; 73,3 \%)$, apresentando diferença estatisticamente significativa $(p<0,0001)$. Nossos achados revelam que o comportamento imitativo é um dos fatores que podem levar o indivíduo a adotar o comportamento de uso de drogas.

Palavras-chave: Adolescência; Drogas; Comportamento Imitativo.

\begin{abstract}
This research investigated the influence that the imitative behavior exercises on the adoption of behaviors related to the use of drugs in adolescents between 12 and 17 years. It is characterized as an observational and cross-sectional with a quantitative approach and it was conducted with 128 adolescents from two public schools in Criciúma/SC. The findings showed that many teenagers have a friend who uses psychoactive substances and started to use of psychoactive substances by influence of these friendships. Regarding to the consumption of alcohol, $35.4 \%$ of students stated that they have made consumed by the influence of friends, being more predominant in male individuals; and $15.6 \%$ said they used other drugs for the same reason. We observed a greater influence of friends in alcohol consumption between 15 and 17 years $(73.3 \%)$, a statistically significant difference $(p<0.0001)$. Our findings reveal that the imitative behavior is one of the factors that can lead a person to adopt the behavior of drug use.
\end{abstract}

Keywords: Adolescence; Drugs; Imitative Behavior. 


\section{INTRODUÇÃO}

Situações de vulnerabilidade na adolescência podem ser desencadeadas por muitos fatores, e dentre estes, o uso e abuso de substâncias psicoativas se configura como o principal gerador desse tipo de situação ${ }^{1}$. Associado a isso temos o problema do tráfico, que representa uma séria ameaça à estabilidade social de um país, sem mencionar os casos relacionados a acidentes de trânsito, suicídio, gravidez na adolescência e outros danos relacionados².

Os serviços de saúde sofrem o impacto causado pelo consumo de drogas, precisando adequar-se a essa demanda. $\mathrm{O}$ número de leitos nos hospitais gerais para tratamento de drogaditos revela uma preocupação dos profissionais e das instituições de saúde que se sentem despreparados e receosos de lidar com usuários em tratamento ${ }^{3}$.

Nesse contexto, a Pesquisa Nacional de Saúde do Escolar (PeNSE) ${ }^{4}$ levantou alguns dados referentes ao uso de álcool nas capitais brasileiras e no Distrito Federal e revelou que $71,4 \%$ dos escolares do nono ano do Ensino Fundamental já experimentaram bebida alcoólica alguma vez na vida, enquanto que $27,3 \%$ a consumiam regularmente. A pesquisa mostrou ainda que a forma mais comum para adquirir bebida alcoólica foi em festas (39,8\%), seguida da compra em mercado, loja, supermercado ou bar (18,4\%). Dados nacionais ${ }^{5}$ mostraram que $13 \%$ dos adolescentes têm padrão intenso de consumo de álcool, e outros 10,0\% consomem álcool de 1 a 3 vezes por mês, com a possibilidade de consumir quantidades arriscadas, sem associação com o gênero. Com relação ao número de internações decorrentes do uso de drogas, alguns levantamentos epidemiológicos têm revelado um aumento gradual até 2003 (de 143.199 internações em 2001 para 145.070 em 2003), com diminuição progressiva de 2004 (134.573) a 2006 (129.619) e com um posterior aumento em 2007 (134.674) ${ }^{5}$.

De acordo com a literatura, o uso de substâncias psicoativas representa um fenômeno multideterminado, ou seja, os fatores relacionados ao uso de substâncias por parte dos adolescentes são variados ${ }^{6}$. No período da adolescência, as várias operações psíquicas atuantes fazem com que o comportamento de usar drogas seja 


\section{Artigo Original}

\section{Saúde e Processos Psicossociais}

algo relativamente comum nessa fase ${ }^{7,8}$, incluindo o uso experimental, esporádico e abusivo ${ }^{9}$.

A manifestação desse tipo de comportamento pode ser aprendido por meio da experiência direta e da observação, as quais acabam por condicionar tal comportamento ${ }^{10}$. Bandura e Peotta ${ }^{11}$ foram os teóricos que explicitaram bem esse fenômeno e além destes autores, algumas pesquisas realizadas nos Estados Unidos comprovaram que o comportamento pode ser aprendido pelos adolescentes por meio da influência parental no uso de cigarro ${ }^{12-14}$.

Conhecer os fatores associados ao uso e abuso de drogas torna-se relevante a partir do momento que permite que intervenções comportamentais sejam pensadas nesse contexto, o que poderia minimizar ou até mesmo inibir esse comportamento deletério associado ao uso de substâncias psicoativas lícitas e ilícitas ${ }^{7}$.

Sendo assim, segundo a linha de pesquisa proposta por Bandura ${ }^{11}$ e tendo por base as considerações levantadas, esse estudo tem como objetivo pesquisar a influência que o comportamento imitativo das amizades exerce num grupo de adolescentes estudantes do ensino médio e fundamental de duas escolas do sul de Santa Catarina.

\section{METODOLOGIA}

Trata-se de um estudo observacional e transversal, de abordagem quantitativa. A população alvo da investigação foi constituída por adolescentes, na faixa etária de 12 a 17 anos, matriculados nos turnos vespertino e noturno de duas escolas da rede pública, localizados no centro do município de Criciúma-SC.

Em uma escola, foram coletados os dados dos escolares do ensino fundamental séries finais ( $8^{\circ}$ e $9^{\circ}$ ano), do período vespertino; e na outra foram coletados os dados dos escolares do ensino médio ( $1^{\circ}$ e $2^{\circ}$ ano), do período noturno. Essas escolas foram escolhidas por disponibilizarem as turmas nos períodos disponíveis para a pesquisa e pela localização geográfica, área central da cidade. Para a seleção das escolas adotou-se o critério da disponibilidade das turmas para a pesquisa e da localização geográfica das escolas. 
Foram incluídos no estudo os adolescentes regularmente matriculados na unidade escolar e frequentando as séries finais do ensino fundamental ( $8^{\circ}$ e $9^{\circ}$ ano) e ensino médio ( $1^{\circ}$ e $2^{\circ}$ ano) das escolas públicas estaduais de Criciúma-SC. Assim, participaram da pesquisa 89 alunos que estavam cursando o ensino fundamental e 39 cursando o ensino médio nas escolas da rede pública citadas, totalizando uma amostra de 128 indivíduos.

A coleta de dados foi iniciada explicando-se aos alunos a proposta do estudo, solicitando-se sua participação voluntária, sendo realizada pelo pesquisador responsável pelo projeto. A coleta foi realizada no período vespertino na escola de ensino fundamental e no período noturno na escola de ensino médio, durante 0 período de aula. Os dados foram obtidos através de um questionário autorrespondido realizado coletivamente e sem limite de tempo para o término do preenchimento. A fim de garantir o sigilo das respostas os nomes dos alunos não foram identificados e o preenchimento dos dados foi realizado sem a supervisão direta de um professor.

As variáveis investigadas foram: sexo (feminino/masculino), faixa etária (11 a 17 anos), uso de drogas lícitas (quantidade ingerida, idade que ingeriu) e ilícitas (experimento, uso mais que uma vez). Por drogas lícitas entende-se aqui como cigarro e/ou álcool, e por drogas ilícitas como maconha, crack, cocaína ou outro tipo de droga.

Especificamente os entrevistados foram questionados sobre o fato de possuírem algum amigo do seu círculo de amizades que já havia usado ou estava usando álcool ao ponto de causar algum problema em casa, no trabalho ou com amigos. Ainda nesse contexto, os alunos foram questionados se foram influenciados a fazer uso de bebidas alcoólicas pela observação do comportamento de beber desses amigos. Outra questão abordou a idade que tiveram essa experiência e a quantidade ingerida nessa ocasião, sendo necessário responder sobre a ingestão de um, dois, três ou mais de quatro copos.

A fim de obter informações sobre o uso de drogas ilícitas, o procedimento foi o mesmo. Assim, os estudantes foram questionados se possuíam algum amigo do círculo de amizades que fez ou estava fazendo uso de algum tipo de droga ilícita e se houve a repetição desse comportamento por meio da observação do comportamento desses amigos. Ainda nesse assunto, foram questionados também 


\section{Artigo Original}

\section{Saúde e Processos Psicossociais}

se esse uso foi restringido somente à experimentação ou se houve repetição em outras oportunidades.

Após a coleta de dados, foi elaborado um banco de dados em planilhas do software Microsoft Excel versão 2012. Em seguida, o banco de dados foi exportado para o software Statistical Package for the Social Sciences (SPSS) versão 20.0, onde foi realizada a análise estatística.

Foram construídos gráficos e tabelas para uma melhor organização e apresentação dos dados, e calculadas algumas medidas descritivas, como média e desvio padrão para as variáveis quantitativas, e frequência absoluta e relativa para as qualitativas. Os cálculos analíticos foram realizados com o nível de significância $\alpha$ $=0,05$ e um intervalo de confiança de 95,0\%. A investigação da existência de associação entre as variáveis qualitativas, como por exemplo, sexo e escolaridade, foi realizada através da aplicação do teste qui-quadrado de associação ou independência, e nas tabelas de contingência foi usada a correção de Yates.

No que consta aos ditames éticos, foi elaborado o termo de consentimento informado, apresentado a cada sujeito da pesquisa e colhida sua assinatura e a assinatura dos pais no caso dos menores de 18 anos. O projeto foi aprovado pelo Comitê de Ética em Pesquisa da Universidade do Extremo Sul Catarinense, sob o protocolo número 406.457/2013.

\section{RESULTADOS}

Foram entrevistados 128 estudantes, sendo que a maioria dos indivíduos foi composta pelo gênero feminino, representando $52,3 \%(n=67)$, com idade média de 16 anos $( \pm 1,72)$. A maioria dos estudantes que participaram do estudo estava cursando o ensino fundamental $60,5 \% \quad(n=89)$. O perfil descritivo da amostra encontra-se disponível na Tabela 1. 
Tabela 1. Perfil descritivo.

\begin{tabular}{cc}
\hline Variável & $\mathbf{n}(\%)$ \\
\hline Sexo & $61(47,7)$ \\
Masculino & $67(52,3)$ \\
Feminino & \\
Série & $42(32,8)$ \\
$8^{\underline{a}}$ ano & $47(36,7)$ \\
$9^{a}$ ano & $39(30,5)$ \\
1o ensino médio & \\
Idade & \\
11 & $5(3,9)$ \\
12 & $28(21,9)$ \\
13 & $29(22,7)$ \\
14 & $28(21,9)$ \\
15 & $6(4,7)$ \\
16 & $21(16,4)$ \\
17 & $11(8,6)$ \\
\hline
\end{tabular}

Fonte: Próprio Autor

Com relação ao uso de álcool por algum amigo do círculo de amizades, $32,3 \%(n=41)$ dos entrevistados relataram ter algum amigo que fez ou faz uso de álcool, chegando a ponto de cair, causar problemas em casa ou com amigos. $O$ restante, $64,6 \%(n=82)$, relatou não ter nenhum amigo que fizesse uso e tenha chegado a essa condição. Ainda sob esse aspecto, observou-se que dentre os que relataram o uso, houve uma predominância do gênero masculino ( $n=22 ; 53,7 \%$ ); e dentre os entrevistados que não informaram tal hábito, a maioria foi do gênero feminino $(n=47 ; 54,7 \%)$.

Quando questionados a respeito do uso de álcool mediante a influência dos pares, 35,4\% ( $n=45)$ dos entrevistados relataram usar o álcool quando estavam na presença de amigos. Na análise estratificada por sexo, observou-se predomínio do 


\section{Artigo Original}

\section{Saúde e Processos Psicossociais}

gênero masculino, representado por $60,0 \%,(n=27)$ dos entrevistados, enquanto que $40,0 \%(n=18)$ dos indivíduos do gênero feminino relataram terem feito o uso. Dessa forma, apesar dos dados sugerirem associação, não houve significância estatística $(p=0,070)$ para o uso de álcool sob influência dos pares quando comparados em relação ao sexo do indivíduo, sugerindo uma associação entre gênero e influência dos amigos para o uso de álcool, conforme ilustra a Tabela 2.

Tabela 2. Características da amostra estratificada por sexo.

\begin{tabular}{|c|c|c|c|}
\hline \multirow[b]{2}{*}{ Variável } & \multicolumn{2}{|c|}{ Sexo } & \multirow[b]{2}{*}{$\mathbf{P}$} \\
\hline & $\begin{array}{l}\text { Masculino } \\
\mathrm{n}(\%)\end{array}$ & $\begin{array}{c}\text { Feminino } \\
\mathrm{n}(\%)\end{array}$ & \\
\hline \multicolumn{4}{|c|}{ Uso de álcool por amigos } \\
\hline Sim & $22(53,7)$ & $19(46,3)$ & 0,492 \\
\hline Não & $39(45,3)$ & $47(54,7)$ & \\
\hline \multicolumn{4}{|l|}{$\begin{array}{l}\text { Influência do uso de } \\
\text { álcool por amigos }\end{array}$} \\
\hline Sim & $27(60,0)$ & $18(40,0)$ & 0,070 \\
\hline Não & $34(41,5)$ & $48(58,5)$ & \\
\hline \multirow{2}{*}{\multicolumn{4}{|c|}{$\begin{array}{l}\text { Quantidade de álcool } \\
\text { ingerida por influência de } \\
\text { amigos }\end{array}$}} \\
\hline & & & \\
\hline Em branco & $9(33,3)$ & $18(66,7)$ & 0,100 \\
\hline Nunca ingeriu & $24(43,6)$ & $31(56,4)$ & \\
\hline 1 a 2 copos & $10(76,9)$ & $3(23,1)$ & \\
\hline 3 a 4 copos & $7(58,3)$ & $5(41,7)$ & \\
\hline 5 ou mais copos & $11(52,4)$ & $10(47,6)$ & \\
\hline
\end{tabular}

Fonte: Próprio Autor

Ainda nessa questão, na análise da faixa etária, observamos maior influência dos amigos no consumo de álcool entre os 15 e 17 anos $(n=33 ; 73,3 \%)$, 
Artigo Original

Saúde e Processos Psicossociais

apresentando diferença estatisticamente significativa $(p<0,0001)$ em relação às demais categorias (Tabela 3). E, ainda com relação à faixa correspondente dos 15 aos 17 anos, a quantidade de álcool ingerida nessas situações mais citada pelos entrevistados foi de 3 a 4 copos ( $n=20 ; 95,2 \%$ ), conforme apresenta a Tabela 3.

Tabela 3. Características da amostra estratificada por faixa etária.

\section{Faixa etária}

\begin{tabular}{ccccc}
\hline Variável & $\begin{array}{c}11-12 \\
\text { anos }\end{array}$ & $\begin{array}{c}13-14 \\
\text { anos }\end{array}$ & $\begin{array}{c}15-17 \\
\text { anos }\end{array}$ & $P$ \\
& & &
\end{tabular}

\section{Uso de álcool por}

amigos

$\begin{array}{cccc}\text { Sim } & 0(0) & 11(26,8) & 30(73,2) \\ \text { Não } & 32(37,2) & 46(53,5) & 8(9,3)\end{array}$

Influência do uso de

álcool por amigos

Sim

Não

$31(37,8)$

$11(24,4)$

$46(56,1)$

$33(73,3)$

$5(6,1)$

\section{Quantidade de álcool}

ingerida por influência

de amigos

Em branco

Nunca ingeriu

1 a 2 copos

3 a 4 copos

5 ou mais copos

$12(44,4)$
$20(36,4)$
$1(7,7)$
$0(0,0)$
$0(0,0)$

$13(48,1)$

$2(7,4)$

$3(5,5)$

$32(58,2)$

$8(61,5)$

$4(30,8)$

$5(41,7)$

$7(58,3)$

$20(95,2)$

\section{Idade que fez uso de \\ álcool por influência de \\ amigos}

Em branco 
Artigo Original

Saúde e Processos Psicossociais

Faixa etária

\begin{tabular}{cccc}
\hline Variável & $\begin{array}{c}\mathbf{1 1 - 1 2} \\
\text { anos }\end{array}$ & $\begin{array}{c}\mathbf{1 3 - 1 4} \\
\text { anos }\end{array}$ & $\begin{array}{c}\mathbf{1 5 - 1 7} \\
\text { anos }\end{array}$ \\
\hline 11 & $1(100)$ & $0(0,0)$ & $0(0,0)$ \\
12 & $1(100)$ & $0(0,0)$ & $0(0,0)$ \\
13 & $0(0,0)$ & $2(100)$ & $0(0,0)$ \\
14 & $0(0,0)$ & $6(100)$ & $0(0,0)$ \\
15 & $0(0,0)$ & $5(62,5)$ & $3(37,5)$ \\
16 & $0(0,0)$ & $0(0,0)$ & $9(100)$ \\
17 & $0(0,0)$ & $0(0,0)$ & $17(100)$ \\
18 & $0(0,0)$ & $0(0,0)$ & $4(100)$
\end{tabular}

Fonte: Próprio Autor

No que se refere ao uso de drogas, tanto lícitas quando ilícitas, excluindo-se o álcool, 40,6\% ( $n=52)$ dos entrevistados relatou que algum amigo já havia feito uso ou estava usando drogas. Da amostra total, verificou-se que $15,6 \%$ ( $n=18)$ dos adolescentes relataram terem feito o uso por influência exercida pelos amigos. $\mathrm{Na}$ amostra estratificada por sexo, verificou-se que a maioria desses estudantes que relataram o uso pertencia ao gênero feminino $(n=3 ; 60,0 \%)$. Quando a amostra foi comparada com a faixa etária, os estudantes dos 13 aos 14 anos e dos 15 aos 17 anos tiveram a mesma proporção ( $n=2 ; 40 \%)$.

Quando questionados a respeito da quantidade de vezes que fizeram uso, $3,9 \%(n=5)$ dos entrevistados disseram ter experimentado apenas nessa ocasião, enquanto que a mesma proporção $(n=5 ; 3,9 \%)$ afirmou ter feito uso mais de uma vez em outras oportunidades.

\section{DISCUSSÃO}

Nosso estudo teve como objetivo analisar a influência do comportamento imitativo dos amigos no uso de substâncias psicoativas em adolescentes. 
A idade média dos alunos que consumiram bebida alcoólica foi de 12 anos, dados que são compatíveis com os dados obtidos em uma pesquisa realizada em 2004 com 1990 adolescentes brasileiros, a qual revelou que a idade média para o consumo foi de 12,5 anos ${ }^{15}$. Uma pesquisa realizada por Malta et al. ${ }^{16}$ mostrou que a experimentação de álcool apresenta maior prevalência no período que vai dos 12 aos 13 anos, sendo a maior prevalência no gênero feminino. Dados do PeNSE ${ }^{4}$ mostraram que $50,0 \%$ dos adolescentes entrevistados referiram ter consumido álcool até os 12 anos de idade. Com base nestas informações percebe-se que grande parte dos adolescentes experimenta a droga quando ainda está no ensino fundamental, o que alerta para a precocidade com que estão tendo contato com as drogas, pois conforme Rodrigues, Batista e Werner ${ }^{8}$ e Soares e Gonçalves Junior, quanto mais cedo o indivíduo começa a fazer uso de tais substâncias, mais elevados são os riscos de dependência, de transtornos mentais associados e de comportamento alterado.

No Brasil a venda de bebidas alcoólicas só é legalmente permitida após os 18 anos de idade, o que nos leva a refletir sobre o contrassenso existente no meio social e acadêmico brasileiro, onde o consumo de bebidas alcoólicas torna-se uma prática comum no ambiente domiciliar, festividades e ambientes públicos, enquanto existe uma lei vigente que proíbe a venda de bebidas alcoólicas a menores de idade ${ }^{17}$. Os adolescentes têm acesso relativamente fácil às drogas, o que leva muitos a beberem em quantidades perigosas, cujo efeito pode resultar numa série de problemas ${ }^{5}$ e em consequências negativas, refletindo em problemas nos estudos, problemas sociais, prática de sexo sem proteção e/ou sem consentimento, maior risco de suicídio ou homicídio e acidentes relacionados ao consumo ${ }^{18}$.

A ingestão de 1 a 2 doses foi mais prevalente no gênero feminino, representando $59,0 \%$ dos casos, resultado semelhante ao encontrado na pesquisa realizada por Malta et al. ${ }^{16}$, que também teve uma predominância do gênero feminino nessa questão, porém com uma proporção menor $(29,9 \%)$.

De acordo com a literatura internacional, a ingestão de 5 doses ou mais para homens e 4 ou mais para as mulheres num único episódio é denominada de beber em "binge", que é uma expressão que indica um estado de consumo de risco ${ }^{5}$. Nessa perspectiva, um estudo transversal realizado em 2009 com amostra de conglomerados de 60.973 estudantes do nono ano do Ensino Fundamental de 


\section{Artigo Original \\ Saúde e Processos Psicossociais}

escolas públicas e privadas das capitais dos estados brasileiros e do Distrito Federal revelou que o consumo de mais de cinco doses foi significativamente maior entre os meninos $(20,1 \% \text { versus } 15,2 \%)^{16}$. O Levantamento Nacional sobre Padrões de Consumo de Álcool ${ }^{5}$ num estudo realizado com 661 adolescentes brasileiros revelou que $13,0 \%$ do total dos adolescentes (17,0\% para os meninos) apresenta padrão intenso de consumo de álcool. Os dados obtidos na nossa pesquisa mostram que a maioria dos que fizeram uso do álcool nessa intensidade foi do gênero masculino $(52,4 \%)$, porém, não existindo diferença estatisticamente significativa no consumo de álcool em relação ao gênero.

Uma pesquisa realizada com 568 adolescentes da cidade de Ribeirão Preto mostrou que dentre os participantes, 14,9\% afirmaram que os responsáveis pelo início do consumo de substâncias psicoativas foram seus amigos ${ }^{6}$, e outro estudo realizado com 300 adolescentes da cidade de Cajazeiras (PB) mostrou que 18,8\% dos entrevistados afirmaram que bebem por influência dos colegas ${ }^{19}$. Nossa casuística revelou resultados diferentes no que concerne à influência exercida pelos amigos, prevalente em 35,4\% $(n=45)$ da amostra, índice maior se comparado aos estudos anteriores.

São muitos os fatores que podem contribuir e motivar o uso de substâncias psicoativas, mas o fator que é mais frequentemente associado ao uso nessa fase da vida é a utilização pelos amigos ${ }^{20}$. Conforme os dados mostram, a influência exercida pelos amigos assume uma importância fundamental no uso de substâncias psicoativas, resultado que corrobora com Pechansky, Szobot e Scivoletto ${ }^{17}$, que, a partir de um estudo que teve a finalidade de descrever aspectos epidemiológicos, etiopatogênicos e diagnósticos associados ao consumo de bebidas alcoólicas por adolescentes, constataram que a experimentação inicial se dá pelo fato de o adolescente ter amigos que usam drogas, gerando uma pressão de grupo na direção do uso ${ }^{17}$.

Assim, podemos inferir que os adolescentes socialmente aceitos por seus pares recebem reforço, acabando por melhorar sua adaptação na área social, pessoal e escolar, o que nos leva a considerar o consumo de bebidas alcoólicas e outras drogas como o aspecto central nessa relação ${ }^{21}$. E esse modelo oferecido pelo grupo de amigos está relacionado com a pressão social exercida em um período do 


\section{Artigo Original \\ Saúde e Processos Psicossociais}

desenvolvimento humano que é particularmente sugestionável e influenciável pela opinião dos pares, gerando tais comportamentos ${ }^{6,19,22}$.

Analisando o aspecto da adoção do comportamento a partir da influência dos amigos, Wang et al. ${ }^{14}$ pontuam que várias teorias do comportamento humano foram utilizadas como base para delinear os fatores determinantes para a aquisição do hábito de fumar, sendo o modelo mais promissor na explicação desse fenômeno o da aprendizagem social, pelo qual ocorre uma interação recíproca entre o comportamento do indivíduo e o ambiente. Sob esse aspecto, Bandura ${ }^{11}$ sugere que nas relações sociais possa ser observado que o comportamento de uma pessoa exerce certo grau de controle sobre o comportamento das outras pessoas, ou seja, os fenômenos da aprendizagem que são resultado da experiência direta tem possibilidade de ocorrer sob base vicária por meio da observação do comportamento de outras pessoas e de suas consequências.

Os estudos realizados com a temática relacionada a drogas raramente dão destaque às amizades entre os jovens como protetoras, pois em geral as intervenções são focadas na influência negativa que as amizades exercem, mas é importante considerar que os grupos de amigos com objetivos e expectativas de realização de vida têm papel fundamental numa etapa em que as influências dos pares são cruciais ${ }^{9}$.

Nosso estudo, bem como outros apontados, demonstrou que o álcool e outras drogas constituem-se como um problema de Saúde Pública no Brasil, necessitando, dessa forma, de intervenções por parte da sociedade e de todas as esferas de governo ${ }^{5}$. Essas intervenções devem ser baseadas no fato de que os adolescentes de determinada comunidade e, no caso, das escolas pesquisadas, não constituem uma massa homogênea de interesses, antes disso, se constituem como um conjunto de individualidades em interação que de forma alguma está isenta de conflitos e contradições ${ }^{23}$, ou seja, devem ser assistidos a partir de seu contexto social, familiar, que é permeado pela construção histórica de sua identidade enquanto adolescentes.

Apesar do fato de que a maioria dos jovens que bebem pesadamente são propensos a reduzir a quantidade de álcool ingerida e passar a beber moderadamente quando chega à fase adulta, não deixa de ser preocupante a conduta de beber na adolescência, já que os que possuem tal hábito estão mais 


\section{Artigo Original}

\section{Saúde e Processos Psicossociais}

propensos a sofrerem as consequências ${ }^{24}$. Nossos achados referentes ao uso de substâncias psicoativas e a influência exercida pelos amigos ressaltam a necessidade de implantar políticas públicas com foco no público adolescente a partir de uma abordagem integrada com ações de caráter preventivo ${ }^{25}$. E essa prevenção deve ser pensada como sinônimo de vida saudável, um processo de grande importância para os jovens que deve incluir a família, a escola, o grupo de pares, a comunidade e a mídia 9 .

\section{CONCLUSÃO}

A população predominante em nosso estudo foi composta de adolescentes do gênero feminino, estudantes do ensino fundamental de duas escolas públicas do município de Criciúma - SC, na faixa etária correspondente dos 13 aos 14 anos de idade. Grande parte dos entrevistados possuía algum amigo do círculo de amizades que fez uso de álcool ou outras drogas, e destes, quase todos foram influenciados a fazer o uso, principalmente no que se refere ao uso de álcool, cujo número máximo de doses ingeridas citado foi maior que 5 .

Diante de nossos achados, pode-se concluir que a adolescência pode ser considerada como um período que torna o jovem mais vulnerável ao uso de substâncias psicoativas, e os dados relativos ao uso nessa fase se mostram preocupantes, mostrando assim a urgência e a importância de aprofundar e fazer investigações nesta área.

Nossa pesquisa contribuiu no sentido de discutir sobre um dos motivos que levam os adolescentes a adotar o comportamento de usar substâncias psicoativas e o papel exercido pelos amigos nesse sentido, que é o comportamento imitativo. Mediante os estudos prévios aqui apresentados, tal influência pode ser exercida pelo círculo de amigos, tendo em vista o fácil acesso às drogas, principalmente o álcool e a pressão exercida no grupo, no qual o sentimento de pertencimento é um fator importante e a influência exercida nesse contexto é fortalecida ainda mais.

Tal discussão abre caminho para a implementação de intervenções eficientes que levem em conta as características inerentes da adolescência, sobretudo dos adolescentes inseridos nesse contexto social, que necessitam da implementação de intervenções que tenham por base o desenvolvimento de 


\section{Artigo Original}

\section{Saúde e Processos Psicossociais}

habilidades sociais, estabelecendo assim um caráter preventivo à ação ao minimizar os efeitos deletérios à saúde e as consequências causadas pelo uso de substâncias psicoativas na adolescência.

Uma possível limitação de nosso estudo deu-se pelo limitado tamanho da amostra, assim, futuras pesquisas podem considerar uma quantidade maior de participantes. Para melhor detalhamento do estudo, o tipo de substância utilizada pelos adolescentes pode também ser investigado em futuros estudos para melhor traçar o perfil de indivíduos que fazem uso abusivo, esporádico ou experimental e, assim, estabelecer uma possível relação entre o tipo de substância usado e a influência exercida pelo grupo de amigos.

\section{REFERÊNCIAS}

1. Almeida MM, Oliveira MA, Pinho PH. O tratamento de adolescentes usuários de álcool e outras drogas: uma questão a ser debatida com os adolescentes? Arch clin psychiatry (São Paulo). 2008;35:76-81.

2. MS. O tratamento de adolescentes usuários de álcool e outras drogas: uma questão a ser debatida com os adolescentes? Ministério da Saúde. Brasília: Ministério da Saúde, SENAD.; 2009.

3. Gabatz RIB, Schmidt AL, Terra MG, Padoin SMM, Silva AA, Lacchini AJB. Percepção dos usuários de crack em relação ao uso e tratamento. Rev gaúch enferm. 2013;34:140-6.

4. IBGE. Pesquisa Nacional de Saúde do Escolar (PeNSE). Rio de Janeiro: IBGE; 2009.

5. Pinsky I, Zaleski M, Laranjeira R, Caetano R. Primeiro levantamento nacional sobre os padrões de consumo de álcool na população brasileira. Rev bras psiquiatr. 2010;32(3):214-5.

6. Pratta EMM, Santos MA. Lazer e uso de substâncias psicoativas na adolescência: possíveis relações. Psicol teor pesqui. 2007;23(1):43-52.

7. Raupp L, Milnitsky-Sapiro C. Adolescência, drogadição e políticas públicas: recortes no contemporâneo. Estud psicol (Campinas). 2009;26:445-54.

8. Soares HLR, Gonçalves HCB, Junior JW. Cérebro e o uso de drogas na infância e adolescência. Fractal rev psicol. 2010;22:639-.

9. Schenker M, Minayo MCS. Fatores de risco e de proteção para o uso de drogas na adolescência. Ciênc saúde coletiva. 2005;10:707-17. 
10. Saúde OMd. The World Health Report, Mental Health: New Understanding, New Hope. Geneva: WHO; 2001.

11. Bandura AP, L. Modificação do comportamento. Rio de janeiro: interamericana; 1979.

12. Krosnick JA, Judd CM. Transitions in social influence at adolescence: Who induces cigarette smoking? Dev psycholDev psychol. 1982;18(3):359.

13. Morgan M, Grube JW. Adolescent cigarette smoking: A developmental analysis of influences. Brit J Dev Psychol. 1989;7(2):179-89.

14. Wang MQ, Fitzhugh EC, Eddy JM, Fu Q, Turner L. Social influences on adolescent's smoking process: a longitudinal analysis. Am j health behav. 1997;21(2):111-7.

15. Campos JADB, Almeida JC, Garcia PPNS, Faria JB. Consumo de álcool entre estudantes do ensino médio do município de Passos - MG. Ciênc saúde coletiva. 2011;16:4745-54.

16. Malta DC, Mascarenhas MDM, Porto DL, Duarte EA, Sardinha LM, Barreto SM, et al. Prevalência do consumo de álcool e drogas entre adolescentes: análise dos dados da Pesquisa Nacional de Saúde Escolar. Rev bras epidemiol. 2011;14:13646.

17. Pechansky F, Szobot CM, Scivoletto S. Uso de álcool entre adolescentes: conceitos, características epidemiológicas e fatores etiopatogênicos. Rev bras psiquiatr. 2004;26:14-7.

18. Galanter M. Recent developments in alcoholis. New York: Kluwer AcademicPlenum Publisher; 2005.

19. Cerqueira G, Lucena C, Gomes A, Freitas A, Rocha N, Mariz S. Consumo de álcool entre estudantes de uma escola pública da cidade de Cajazeiras, PB. SMAD, Rev eletrônica saúde mental alcool drog. 2011;7(1):18-24.

20. Micheli D, Formigoni ML. As razões para o primeiro uso de drogas e as circunstâncias familiares prevêem os padrões de uso futuro. J Bras Dependência Química. 2001;2(1):20-30.

21. Silva SED, Padilha MI. Atitudes e comportamentos de adolescentes em relação à ingestão de bebidas alcoólicas. Rev Esc Enferm USP. 2011;45:1063-9.

22. Lima I, Paliarin M, Zaleski E, Arantes S. História oral de vida de adolescentes dependentes químicos, internados no setor de psiquiatria do hospital regional de Mato Grosso do Sul para tratamento de desintoxicação. SMAD, Rev eletrônica saúde mental alcool drog. 2008;4(1):01-11. 


\section{Artigo Original}

Saúde e Processos Psicossociais

23. Schraiber LB, Nemes MIB, Mendes-Gonçalves RB. Saúde do adulto: programas e ações na unidade básica. São Paulo: Editora Hucitec; 2000.

24. Martins R, Cruz L, Teixeira $P$, Manzato A. Padrão de consumo de álcool entre estudantes do ensino médio de uma cidade do interior do estado de São Paulo. SMAD, Rev eletrônica saúde mental alcool drog. 2008;4(1):01-16. 